\title{
Memetic Multilevel Hypergraph Partitioning
}

\author{
Robin Andre, Sebastian Schlag and Christian Schulz \\ Karlsruhe Institute of Technology, Karlsruhe, Germany \\ robin.andreeira.uka.de \{ sebastian.schlag, christian.schulz\}@ @it.edu \\ University of Vienna, Vienna, Austria \\ christian.schulzlanivie.ac.at
}

\begin{abstract}
Hypergraph partitioning has a wide range of important applications such as VLSI design or scientific computing. With focus on solution quality, we develop the first multilevel memetic algorithm to tackle the problem. Key components of our contribution are new effective multilevel recombination and mutation operations that provide a large amount of diversity. We perform a wide range of experiments on a benchmark set containing instances from application areas such VLSI, SAT solving, social networks, and scientific computing. Compared to the stateof-the-art hypergraph partitioning tools hMetis, $\mathrm{PaToH}$, and KaHyPar, our new algorithm computes the best result on almost all instances.
\end{abstract}




\section{Introduction}

Given an undirected hypergraph $H=(V, E)$, the $k$-way hypergraph partitioning problem is to find disjoint subsets of its vertex set, $V_{1}, \ldots, V_{k}$, called blocks, such that the blocks have roughly equal size and an objective function involving the cut hyperedges is minimized, e.g. , the sum of the weights of those hyperedges that connect multiple blocks. The hypergraph partitioning problem has many important applications in practice such as scientific computing or VLSI design [44]. In particular VLSI design is a field where small improvements can lead to significant savings [60]. Hence, our focus in this work is on solution quality.

It is well known that the hypergraph partitioning problem (HGP) is NP-hard [40] so that mostly heuristic algorithms are used in practice. A successful heuristic to partition large hypergraphs is the multilevel approach [16]. Here, the hypergraph is recursively contracted to obtain smaller hypergraphs which should reflect the same basic structure as the input. After applying an initial partitioning algorithm to the smallest hypergraph, contraction is undone and, at each level, a local search method is used to improve the partitioning induced by the coarser level. The intuition behind this approach is that a good partition at one level of the hierarchy will also be a good partition on the next finer level. Hence, depending on the definition of the neighborhood, local search algorithms are able explore local solution spaces very effectively in this setting. However, they are also prone to get stuck in local optima [36]. The multilevel paradigm helps to some extent, since local search has a more global view on the problem on the coarse levels and a very fine-grained view on the fine levels of the multilevel hierarchy. In addition, as with many other metaheuristics, multilevel HGP gives better results if several repeated runs are made with some measures taken to diversify the search.

Still even a large number of repeated executions can only scratch the surface of the huge space of possible partitionings. In order to explore the global solution space extensively we need more sophisticated metaheuristics. This is where memetic algorithms (MAs), i.e., genetic algorithms combined with local search [37], come into play. Memetic algorithms allow for effective exploration (global search) and exploitation (local search) of the solution space. The general idea behind genetic algorithms is to use mechanisms inspired by biological evolution such as selection, mutation, recombination and survival of the fittest. A genetic algorithm (GA) starts with a population of individuals (in our case partitions of the hypergraph) and evolves the population over several generational cycles (rounds). In each round, the GA uses a selection rule based on the fitness of the individuals of the population to select good individuals and combines them to obtain improved offspring [29]. When an offspring is generated an eviction rule is used to select a member of the population to be replaced by the new offspring. For an evolutionary algorithm it is of major importance to preserve diversity in the population [10], i.e., the individuals should not become too similar in order to avoid a premature convergence of the algorithm. This is usually achieved by using mutation operations and by using eviction rules that take similarity of individuals into account.

Several genetic and memetic hypergraph partitioning algorithms have already been proposed in the literature [6778|15|38]. However none of them is considered to be truly competitive with state-of-the-art tools [19]. We believe that this is due to the fact that all of them employ flat (i.e., non-multilevel) partitioning algorithms to drive the exploitation of the local solution space.

Our main contribution in this paper therefore is a technique that integrates a memetic algorithm with a multilevel hypergraph partitioner. To this end, we present sophisticated recombination and mutation operators as well as a replacement rule that uses a problem specific similarity measure. In contrast to previous work [677|8 15[38], which only considered small and outdated [13] ACM/SIGDA benchmark instances [46] (dating back to the late 1980s), we perform extensive experiments on a large benchmark set containing hypergraphs from several application areas. Our experiments indicate that our algorithm is able to compute partitions of very high quality and scales well to large networks. It performs better than KaHyPar, which seems to be the current method of choice among the available hypergraph partitioning tools unless speed 
is more important than quality [30], and the state-of-the-art HGP tools hMetis [35[36] and PaToH [17]. In a setting where competing algorithms get the same fairly large amount of time to compute a solution, our new algorithm computes the best result on 648 out of the 700 benchmark instances. This is in contrast to previous non-multilevel evolutionary algorithms for the problem, which are not considered to be competitive with state-of-the-art tools [19].

\section{Preliminaries}

Notation and Definitions. An undirected hypergraph $H=(V, E, c, \omega)$ is defined as a set of $n$ vertices $V$ and a set of $m$ hyperedges/nets $E$ with vertex weights $c: V \rightarrow \mathbb{R}_{>0}$ and net weights $\omega: E \rightarrow \mathbb{R}_{>0}$, where each net is a subset of the vertex set $V$ (i.e., $e \subseteq V$ ). The vertices of a net are called pins. We extend $c$ and $\omega$ to sets, i.e., $c(U):=\sum_{v \in U} c(v)$ and $\omega(F):=\sum_{e \in F} \omega(e)$. A vertex $v$ is incident to a net $e$ if $v \in e . \mathrm{I}(v)$ denotes the set of all incident nets of $v$. The set $\Gamma(v):=\{u \mid \exists e \in E:\{v, u\} \subseteq e\}$ denotes the neighbors of $v$. The size $|e|$ of a net $e$ is the number of its pins. A $k$-way partition of a hypergraph $H$ is a partition of its vertex set into $k$ blocks $\Pi=\left\{V_{1}, \ldots, V_{k}\right\}$ such that $\bigcup_{i=1}^{k} V_{i}=V, V_{i} \neq \emptyset$ for $1 \leq i \leq k$ and $V_{i} \cap V_{j}=\emptyset$ for $i \neq j$. We use $b[v]$ to refer to the block of vertex $v$. We call a $k$-way partition $\Pi \varepsilon$-balanced if each block $V_{i} \in \Pi$ satisfies the balance constraint: $c\left(V_{i}\right) \leq L_{\max }:=(1+\varepsilon)\left\lceil\frac{c(V)}{k}\right\rceil$ for some parameter $\varepsilon$. Given a $k$-way partition $\Pi$, the number of pins of a net $e$ in block $V_{i}$ is defined as $\Phi\left(e, V_{i}\right):=\left|\left\{v \in V_{i} \mid v \in e\right\}\right|$. For each net $e, \Lambda(e):=\left\{V_{i} \mid \Phi\left(e, V_{i}\right)>0\right\}$ denotes the connectivity set of $e$. The connectivity of a net $e$ is the cardinality of its connectivity set: $\lambda(e):=|\Lambda(e)|$. A net is called cut net if $\lambda(e)>1$. The $k$-way hypergraph partitioning problem is to find an $\varepsilon$-balanced $k$-way partition $\Pi$ of a hypergraph $H$ that minimizes an objective function over the cut nets for some $\varepsilon$. Several objective functions exist in the literature [5[40]. The most commonly used cost functions are the cut-net metric cut $(\Pi):=\sum_{e \in E^{\prime}} \omega(e)$ and the connectivity metric $(\lambda-1)(\Pi):=\sum_{e \in E^{\prime}}(\lambda(e)-1) \omega(e)$, where $E^{\prime}$ is the set of all cut nets [24[26]. Optimizing both objective functions is known to be NP-hard [40]. In this paper, we use the connectivity-metric $(\lambda-1)(\Pi)$. Contracting a pair of vertices $(u, v)$ means merging $v$ into $u$. The weight of $u$ becomes $c(u):=c(u)+c(v)$. We connect $u$ to the former neighbors $\Gamma(v)$ of $v$ by replacing $v$ with $u$ in all nets $e \in \mathrm{I}(v) \backslash \mathrm{I}(u)$ and remove $v$ from all nets $e \in \mathrm{I}(u) \cap \mathrm{I}(v)$. Uncontracting a vertex $u$ reverses the contraction.

\subsection{Related Work}

Overview. Driven by applications in VLSI design and scientific computing, HGP has evolved into a broad research area since the 1990s. We refer to [5|11|44|54] for an extensive overview. In the following, we focus on issues closely related to the contributions of our paper. Memetic algorithms (MAs) were introduced in [42] and formalized in [45] as an extension to the concept of genetic algorithms (GAs) [31]. While GAs effectively explore the global solution space, MAs additionally allow for exploitation of the local solution space by incorporating local search methods into the genetic framework. We refer to [43] for an introduction to memetic algorithms. While several genetic and memetic flat (i.e., non-multilevel) hypergraph partitioning algorithms have been proposed in the literature, none of them is considered to be truly competitive with state-of-the-art tools [19]. Well-known multilevel HGP software packages with certain distinguishing characteristics include PaToH [17] (originating from scientific computing), hMetis [35|36] (originating from VLSI design), KaHyPar [2 30 52] (general purpose, $n$-level), Mondriaan [57] (sparse matrix partitioning), MLPart [4] (circuit partitioning), Zoltan [25], Parkway [55], and SHP [34] (distributed), UMPa [56] (directed hypergraph model, multi-objective), and $\mathrm{kPaToH}$ (multiple constraints, fixed vertices) [9]. 
Evolutionary Hypergraph Partitioning. Saab and Rao [47] present an evolution-based approach for solving a $k$-way multi-objective, multi-constraint hypergraph partitioning problem. Since the algorithm only works with one individual, it does not use any recombination operators. Instead, the solution initially generated via bin packing is evolved using a randomized algorithm that moves vertices to different blocks if their gain is greater than some random value. Hulin [33] provides a GA that uses a coding scheme specifically tailored to circuit bipartitioning along with crossover and mutation operations that respect the coding. Bui and Moon [15] present a steady-state MA for ratio cut bipartitioning of hypergraphs, which uses a weak variation of the FM algorithm [28] as local search engine. To improve the performance of the crossover operation, a preprocessing step re-indexes the vertices by the visiting order of a weighted depth first search on the clique-representation [32] of the hypergraph. Areibi [6] present a memetic algorithm that combines a GA with a modified version of Sanchis' $k$-way FM algorithm [48]. Areibi and Yang [7] enhance the MA presented in [6] with a preprocessing step that clusters and contracts vertices to reduce the complexity of the hypergraphs. Furthermore, the initial population contains both random as well as good solutions generated using the GRASP heuristic [27]. Armstrong et al. [8] propose a $k$-way MA that performs crossover, mutation and local search on multiple individuals in parallel. The traditional FM algorithm [28] and Sanchis' $k$ way FM version [48] are used for local search. Kim et al. [38] present a steady-state MA for hypergraph bipartitioning, which uses a modified FM algorithm that works with lock-gains [39]. Note that none of these algorithms makes use of the multilevel paradigm.

Evolutionary Graph Partitioning. We refer to the survey of Kim et al. [37] for a general overview and more material on genetic approaches for graph partitioning. Soper et al. [53] provide the first algorithm that combined an evolutionary search algorithm within a multilevel graph partitioner. Here, crossover and mutation operators compute edge biases based on the input individuals. A similar approach based on perturbations of edge weights has been used by Delling et al. [23]. Benlic et al. [13] provide a multilevel memetic algorithm for balanced graph partitioning. PROBE [18] is a metaheuristic which can be viewed as a genetic algorithm without selection. It outperforms other metaheuristics, but it is restricted to the case $k=2$ and

$\varepsilon=0$. KaHIP [51] contains KaFFPaE [50], which has a general recombine operator framework based on a multilevel algorithm.

\section{$2.2 k$-way Hypergraph Partitioning using KaHyPar}

Since our memetic algorithm builds on top of the KaHyPar framework, we briefly review its core components. While traditional multilevel HGP algorithms contract matchings or clusterings and therefore work with a coarsening hierarchy of $\mathcal{O}(\log n)$ levels, KaHyPar instantiates the multilevel paradigm in the extreme $n$-level version, removing only a single vertex between two levels. Vertex pairs $(u, v)$ to be contracted are determined using the heavy-edge rating function $r(u, v):=\sum_{e \in E^{\prime}} \omega(e) /(|e|-1)$, where $E^{\prime}:=\{\mathrm{I}(u) \cap \mathrm{I}(v)\}$. The coarsening process stops as soon as the number of vertices drops below a certain threshold or no more contractions are possible. The framework currently contains two coarsening algorithms. The first algorithm [52] contracts vertices in decreasing rating score order using a priority queue to store and update the ratings. The second algorithm [2] immediately contracts each vertex with its highest-rated neighbor in random order. After coarsening, a portfolio of simple algorithms is used to create an initial partition of the coarsest hypergraph. During uncoarsening, strong localized local search heuristics based on the FM algorithm [28 48] are used to refine the solution by moving vertices to other blocks in the order of improvements in the optimization objective. The framework provides a recursive bisection algorithm to optimize the cutnet metric (KaHyPar-R [52]) as well as a direct $k$-way algorithm to optimize the $(\lambda-1)$ metric (KaHyPar$\mathrm{K}$ [2]). Recently, Heuer and Schlag [30] integrated an improved coarsening scheme into KaHyPar-K that 
incorporates global information about the structure of the hypergraph into the coarsening process. It uses community detection in a preprocessing step and prevents inter-community contractions during coarsening. This version is referred to as KaHyPar-CA. Unless mentioned otherwise, we use the default configurations provided by the authors 1 .

\section{Memetic Multilevel Hypergraph Partitioning}

We now explain the components of our memetic multilevel hypergraph partitioning algorithm. Given a hypergraph $H$ and a time limit $t$, the algorithm starts by creating an initial population of $\mathcal{P}$ individuals, which in our case correspond to $\varepsilon$-balanced $k$-way partitions of $H$. The population size $|\mathcal{P}|$ is determined dynamically by first measuring the time $t_{\mathrm{I}}$ spend to create one individual. Then $\mathcal{P}$ is chosen such that the time to create $|\mathcal{P}|$ individuals is a certain percentage $\delta$ of the total running time $t:|\mathcal{P}|:=\max (3, \min (50, \delta$. $\left.\left.\left(t / t_{I}\right)\right)\right)$, where $\delta$ is a tuning parameter. The lower bound on the population size is chosen to ensure a certain minimum of diversity, while the upper bound is used to ensure convergence. In contrast to previous approaches [6 8 15]33|38] the population is not filled with randomly generated individuals, but high-quality solutions computed by KaHyPar-CA.

To judge the fitness of an individual we use the connectivity $(\lambda-1)(\Pi)$ of its partition $\Pi$. The initial population is evolved over several generational cycles using the steady-state paradigm [22], i.e., we generate only one offspring per generation. Our two-point and multi-point recombination operators described in Section 3.1 improve the average quality of the population by effectively combining different solutions to the HGP problem. In order to sufficiently explore the global search space and to prevent premature convergence, it is important to keep the population diverse [10]. This becomes even more relevant in our case, since with KaHyPar-CA we use powerful heuristics to exploit the local solution space. Previous work on evolutionary algorithms for HGP [7/8 8 15|33|38] used simple mutations that change the block of each vertex uniformly at random with a small probability. In contrast to these simple, problem agnostic operators, we propose mutation operators based on V-cycles [59] that exploit knowledge of the problem domain and create offspring solutions in the vicinity of the current population. Furthermore in Section 3.3 we propose a replacement strategy which considers fitness and similarity to determine the individual to be evicted from the population.

\subsection{Recombination Operators}

The evolutionary algorithms for HGP presented in Section 2.1 use simple multi-point crossover operators which split the parent partitions into several parts and then combine these parts to form new offspring (see Figure 1 (a)). Since these operators do not take the structure of the hypergraph into account, offspring solutions may have considerably worse fitness than their parents. By generalizing the recombine operator framework presented in [50] from graphs to hypergraphs, our two-point recombine operators described in this section assure that the fitness of the offspring is at least as good as the best of both parents. The edge frequency based multi-point recombination operator described afterwards gives up this property, but still generates good offspring.

Two-Point Recombine. The operator starts with selecting parents for recombination using binary tournament selection (without replacement) [14]. Two individuals $I_{1}$ and $I_{2}$ are chosen uniformly at random from $\mathcal{P}$ and the individual with better fitness (i.e., lower $(\lambda-1)$ objective) becomes the first parent $P_{1}$. This process is then repeated to determine the second parent $P_{2}$. A tournament size of two is chosen to keep the selection pressure low and to avoid premature convergence, since all our individuals already constitute high-quality solutions. Both individuals/partitions are then used as input of a modified multilevel partitioning scheme as

\footnotetext{
1 https://github.com/SebastianSchlag/kahypar/tree/master/config
} 

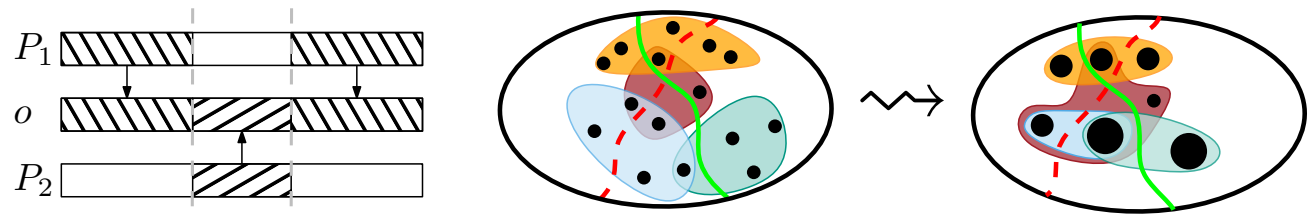

Fig. 1: (a) Traditional, problem agnostic crossover operation to combine parent partitions $P_{1}$ and $P_{2}$ to offspring $o$. (b) Recombination using modified multilevel coarsening to combine two partitions (dashed red line and solid green line). Each cut net $e$ remains in the coarse hypergraph and maintains its connectivity $\lambda(e)$ regarding both partitions.

follows: During coarsening, two vertices $u$ and $v$ are only allowed to be contracted if both parents agree on the block assignment of both vertices, i.e., if $b_{1}[u]=b_{1}[v] \wedge b_{2}[u]=b_{2}[v]$. This is a generalization from multilevel evolutionary GP, i.e. [50], where edges running between two blocks are not eligible for contraction and therefore remain in the graph. In other words, our generalization allows two vertices of the same cut net to be contracted as long as the input individuals agree that they belong to the same block. For HGP, this restriction ensures that cut nets $e$ remain in the coarsened hypergraph and maintain their connectivity $\lambda(e)$ regarding both partitions. This modification is important for our optimization objective, because it allows us to use the partition of the better parent as initial partition of the offspring. Since we can skip the initial partitioning phase and therefore do not need a sufficiently large number of vertices in the coarsest hypergraph to compute a good initial partition [36], we alter the stopping criterion of the coarsening phase such that it stops when no more contractions are possible. The high quality solution of the coarsest hypergraph contains two different classes of vertices: Those for which both parent partitions agree on a block assignment and those for which they don't (see Figure 1 (b) for an example). During the uncoarsening phase, local search algorithms can then use this initial partitioning to (i) exchange good parts of the solution on the coarse levels by moving few vertices and (ii) to find the best block assignment for those vertices, for which the parent partitions disagreed. Since KaHyPar's refinement algorithms guarantee nondecreasing solution quality, the fitness of offspring solutions generated using this kind of recombination is always at least as good as the better of both parents.

Edge-Frequency Multi-Recombine. The operator described previously is restricted to recombine $p=2$ partitions to improved offspring of nondecreasing quality. Sanders and Schulz [50] specifically restrict their operators to this case, arguing that in the course of the algorithm a series of two-point recombine operations to some extend emulates a multi-point recombine. We here present a reasonable multi-point recombine operation to partially evaluate this hypothesis in our experimental evaluation. Our recombine operator uses the concept of (hyper)edge frequency [60] to pass information about the cut nets of the $t$ best individuals in the population on to new offspring. The frequency $f(e)$ of a net $e$ hereby refers to the number of times it appears in the cut in the $t$ best solutions: $f(e):=|\{I \in t \mid \lambda(e)>1\}|$. We use $t=\lceil\sqrt{|\mathcal{P}|}$, which is a common value in evolutionary algorithms [23]. Our multi-recombine operator then uses this information to create a new individual in the following way. The coarsening algorithm is modified to prefer to contract vertex pairs $(u, v)$ which share a large number of small, low-frequency nets. This is achieved by replacing the standard heavy-edge rating function of KaHyPar with the rating function [60] shown in Eq. 1 .

$$
r(u, v):=\frac{1}{c(v) \cdot c(u)} \sum_{e \in\{\mathrm{I}(v) \cap \mathrm{I}(u)\}} \frac{\exp (-\gamma f(e))}{|e|} .
$$


This rating function disfavors the contraction of vertex pairs incident to cut nets with high frequency, because these nets are likely to appear in the cut of high quality solutions. The tuning parameter $\gamma$ is used as a damping factor. After coarsening stops, we compute an initial partition of the coarsest hypergraph using KaHyPar's initial partitioning algorithms and refine it during the uncoarsening and local search phase.

\subsection{Mutation Operations and Diversification}

We define two mutation operators based on V-cycles. All operators are applied to a random individual $I$ of the current population. The main idea of V-cycle based mutation operators is to reuse an already computed partition as input for the multilevel approach and to iterate coarsening and local search phases several times using different seeds for randomization. This approach has been applied successfully in evolutionary GP [50], therefore we also adopt it for HGP. Similar to the recombine operator described in Section 3.1 . the quality of the solution is maintained by only contracting vertex pairs $(u, v)$ belonging to the same block $(b[u]=b[v])$. By distinguishing two possibilities for initial partitioning, we define two different mutation operators: The first one uses the current partition of the individual as initial partition of the coarsest hypergraph and guarantees nondecreasing solution quality. The second one employs KaHyPar's portfolio of initial partitioning algorithms to compute a new solution for the coarsest hypergraph. During uncoarsening, local search algorithms improve the solution quality and thereby further mutate the individual. Since the second operator computes a new initial partition which might be different from the original partition of $I$, the fitness of offspring generated by this operator can be worse than the fitness of $I$.

\subsection{Replacement Strategy}

All recombination and mutation operators create one new offspring $o$. In order to keep the population diverse, we evict the individual most similar to the offspring among all individuals whose fitness is equal to or worse than $o$. Previous work on bipartitioning [15[38] used the Hamming distance as a metric to measure the similarity between partitions. We propose a more sophisticated similarity measure that takes into account the connectivity $\lambda(e)$ of each cut net $e$. For each individual, we compute the multi-set $D:=\{(e, m(e))$ : $e \in E\}$, where $m(e):=\lambda(e)-1$ is the multiplicity (i.e. number of occurrences) of $e$. Thus each cut net $e$ is represented $\lambda(e)-1$ times in $D$. The difference of two individuals $I_{1}$ and $I_{2}$ is the computed as $d\left(I_{1}, I_{2}\right):=\left|D_{1} \ominus D_{2}\right|$, where $\ominus$ is the symmetric difference.

\section{Experimental Evaluation}

System and Methodology. We implemented the memetic algorithm described in the previous section using the latest version of the KaHyPar framework. The code is written in $\mathrm{C}++$ and compiled using $\mathrm{g}++-5.2$ with flags $-03-$ mtune=native - march=native. We refer to the algorithm presented in this paper as EvoHGP. All experiments are performed on a cluster with 512 nodes, where each node has two Intel Xeon E5-2670 Octa-Core (Sandy Bridge) processors clocked at 2.6 GHz, 64 GB main memory, $20 \mathrm{MB}$ L3- and 8x256 KB L2-Cache and runs RHEL 7.4.

We compare EvoHGP with two different configurations of KaHy-Par-CA [30], as well as to the $k$ way (hMetis-K) and the recursive bisection variant (hMetis-R) of hMetis 2.0 (p1) [35[36], and to PaToH 3.2 [17]. These HGP libraries were chosen because they provide the best solution quality [30]2]. The first configuration of KaHyPar-CA corresponds to the default configuration as described in [30]. Since it is known that global search strategies are more effective than plain restarts [49], we augment KaHyPar-CA with Vcycles (in a similar fashion as the first mutation operator) using a maximum number of $100 \mathrm{~V}$-cycle iterations 
per partitioner call. This new enhanced version of KaHyPar-CA constitutes the second configuration and is referred to as KaHyPar-CA-V. hMetis and PaToH are configured as described in [30]. Since PaToH ignores the random seed if configured to use the quality preset, we only report result of the default configuration (PaToH-D). For all five algorithms we perform repeated runs using different random seeds for each run.

To evaluate the impact of different algorithmic components of our algorithm in Section 4.1 each EvoHGP configuration gets two hours time per test instance to compute a solution. For the final evaluation in Section 4.2 all algorithms get eight hours time per test instance. In both cases, we perform five repetitions with different seeds for each test instance and algorithm. Due to the large amount of computing time necessary to perform these experiments, we always partition 16 instances in parallel on a single node. We use the arithmetic mean when averaging over solutions of the same instance and the geometric mean when averaging over different instances in order to give every instance a comparable influence on the final result. In order to compare EvoHGP with the different algorithms, we present two kinds of plots: Convergence plots [50] show the evolution of solution quality over time normalized by instance size, while performance plots [52] are used to compare the best solutions of all algorithms on a per-instance basis.

Convergence Plots. We start by explaining how to compute the data for a single instance $I$, i.e., a $k$-way partition of a hypergraph $H$. Whenever an algorithm computes a partition that improves the solution quality, it reports a pair $(t,(\lambda-1))$, where the timestamp $t$ is the currently elapsed time. For $r$ repetitions with different seeds $s$, these $r$ sequences $T_{s}^{I}$ of pairs are merged into one sequence $T^{I}$ of triples $(t, s,(\lambda-1))$, which is sorted by the timestamp $t$. Since we are interested in the evolution of the solution quality, we compute the sequence $T_{\min }^{I}$ representing event-based average values. We start by computing the average connectivity $\bar{c}$ and the average time $\bar{t}$ using the first pair $(t,(\lambda-1))$ of all $r$ sequences $T_{s}^{I}$ and insert $(\bar{t}, \bar{c})$ into $T_{\min }^{I}$. We then sweep through the remaining entries $(t, s,(\lambda-1))$ of $T^{I}$. Each entry corresponds to a partition computed at timestamp $t$ using seed $s$ that improved the solution quality to $(\lambda-1)$. For each entry we therefore replace the old connectivity value of seed $s$ that took part in the computation of $\bar{c}$ with the new value $(\lambda-1)$, recompute $\bar{c}$ and insert a new pair $(t, \bar{c})$ into $T_{\min }^{I} . T_{\min }^{I}$ therefore represents the evolution of the average solution quality $\bar{c}$ for instance $I$ over time. In a final step, we create the normalized sequence $N_{\min }^{I}$, where each entry $(t, \bar{c})$ in $T_{\min }^{I}$ is replaced by $\left(t_{n}, \bar{c}\right)$ where $t_{n}:=t / t_{I}$ and $t_{I}$ is the average time that KaHyPar-CA needs to compute a $k$-way partition of $H$. Average values over multiple instances are then obtained as follows: All sequences $N_{\min }^{I}$ of pairs $\left(t_{n}, \bar{c}\right)$ are merged into a sequence $N_{\min }$ of triples $\left(t_{n}, \bar{c}, I\right)$, which is then sorted by $t_{n}$. The final sequence $S_{\mathcal{G}}$ presenting event-based geometric averages values is then computed as follows: We start by computing the average normalized time $\overline{t_{n}}$ and the geometric mean connectivity $\mathcal{G}$ over all instances $I$ using the first value of all $N_{\min }^{I}$ and insert $\left(\overline{t_{n}}, \mathcal{G}\right)$ into $S_{\mathcal{G}}$. We then sweep through the remaining entries of $N_{\min }$. For each entry $\left(t_{n}, \bar{c}, I\right)$, we replace the old connectivity value of $I$ that took part in the computation of $\mathcal{G}$ with the new value $\bar{c}$, recompute $\mathcal{G}$ and insert $\left(t_{n}, \mathcal{G}\right)$ into $S_{\mathcal{G}}$. The sequence $S_{\mathcal{G}}$ therefore represents the evolution of the solution quality averaged over all instances and repetitions.

Performance Plots. These plots relate the smallest minimum connectivity of all algorithms to the corresponding connectivity produced by each algorithm on a per-instance basis. For each algorithm, these ratios are sorted in increasing order. The plots use a cube root scale for both axes to reduce right skewness [20] and show $1-$ (best/algorithm) on the y-axis to highlight the instances were each partitioner performs badly. A point close to one indicates that the partition produced by the corresponding algorithm was considerably worse than the partition produced by the best algorithm. A value of zero therefore indicates that the corresponding algorithm produced the best solution. Points above one correspond to infeasible solutions that violated the balance constraint. Thus an algorithm is considered to outperform another algorithm if its corresponding ratio values are below those of the other algorithm. In order to include instances with a cut of zero into the results, we set the corresponding cut values to one for ratio computations. 
Benchmark Instances. We evaluate our algorithm on a representative subset of 100 hypergraphs from the benchmark set of Heuer and Schlag [30] $]^{2}$, which contains instances from four benchmark sets: the ISPD98 VLSI Circuit Benchmark Suite [3], the DAC 2012 Routability-Driven Placement Contest [58], the University of Florida Sparse Matrix Collection [21], and the international SAT Competition 2014 [12]. Sparse matrices are translated into hypergraphs using the row-net model [17], i.e., each row is treated as a net and each column as a vertex. SAT instances are converted to three different representations: For literal hypergraphs, each boolean literal is mapped to one vertex and each clause constitutes a net [44], while in the primal model each variable is represented by a vertex and each clause is represented by a net. In the dual model the opposite is the case [41]. The latter two models are more common in the SAT solving community than the literal model proposed in [44]. All hypergraphs have unit vertex and net weights. An overview of our benchmark sets is given in Tables 2 and 3 in Appendix A To compare EvoHGP with the best competing algorithms, all 100 hypergraphs are partitioned into $k \in\{2,4,8,16,32,64,128\}$ blocks with $\varepsilon=0.03$. For each hypergraph $H$ and each value of $k$, a $k$-way partition $H$ is considered to be one test instance, resulting in a total of 700 instances. In order to save running time, we choose a subset of 25 hypergraphs shown in Table 2, $k=32$, and $\varepsilon=0.03$ to evaluate the impact of different algorithmic components of our algorithm (recombine and mutation operations) before we run the algorithms on the large benchmark set.

\subsection{Influence of Algorithmic Components}

All configurations determine their population size $\mathcal{P}$ dynamically such that $\delta=15 \%$ of the total time is spent to create the initial population. According to the results of Wichlund and Aas [60], the damping factor $\gamma$ used for edge frequency calculations is set to $\gamma=0.5$. We use a naming scheme to refer to different configurations of our algorithm. All configuration names start with EvoHGP followed by abbreviations for the added recombine and mutation operations (multiple abbreviations are used to add multiple operations). Abbreviation $+\mathrm{C}$ refers to EvoHGP using twopoint recombine operations, +ER refers to EvoHGP using multi-recombine operations, and finally $+\mathrm{M}_{x}$ adds mutation operations with a mutation chance of $x$. Whenever a mutation operation is performed, both operators have a 50 percent change of being chosen. Figure 2 compares different configurations of EvoHGP. Of all configurations, EvoHGP+ER, which relies only on multi-

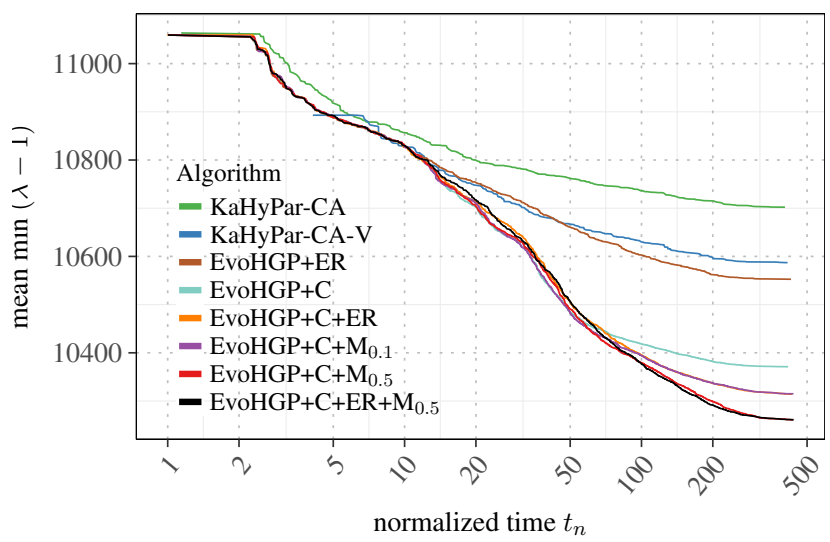

Fig. 2: Influence of EvoHGPs algorithmic components. point recombine operations, performs worst, being only slighthly better than KaHyPar-CA-V. Comparing its performance with EvoHGP+C (which uses only two-point recombine operations), we can see that it is indeed beneficial to guarantee nondecreasing solution quality for combine operations. However combining both recombination operators results in a performance similar to EvoHGP+C+M $+\mathrm{M}_{0.1}$. This can be explained by the fact that multi-recombines also act as mutation operator in that they don't guarantee nondecreasing performance. Due to the fact that the strong multilevel local search engine KaHyPar-CA computes high quality solutions, we see that a significant amount of mutations is necessary to ensure diversity in the population. While EvoHGP $+\mathrm{C}+\mathrm{M}_{0.1}(10 \%$ mutation chance performed best for evolutionary

\footnotetext{
${ }^{2}$ The benchmark set was downloaded from http://algo2.iti.kit.edu/schlag/sea2017/
} 

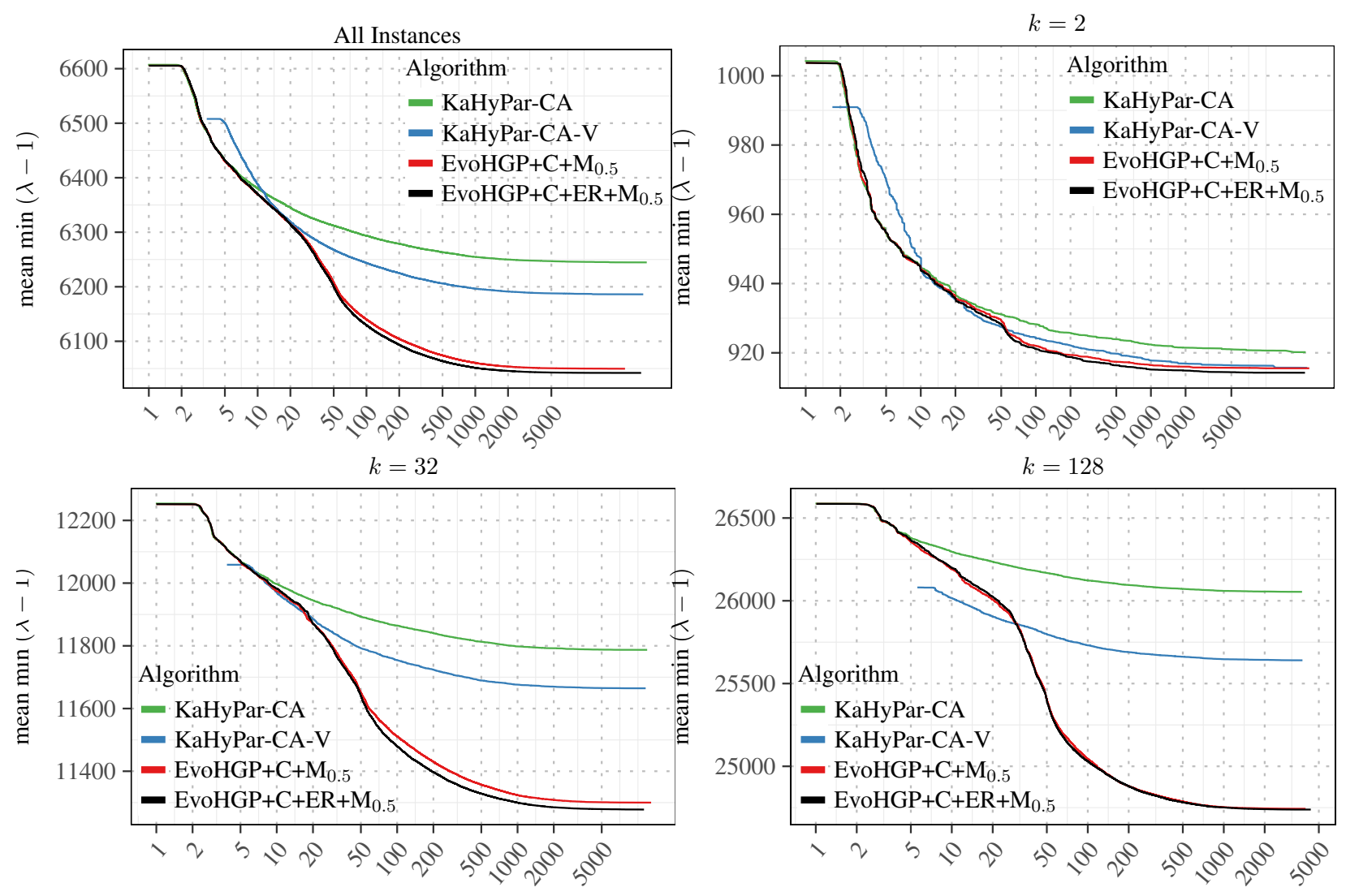

Fig. 3: Convergence plots for all instances and for different values of $k$.

graph partitioning in [50]) performs equally well as EvoHGP+C+ER, increasing the mutation rate to $50 \%$ $\left(\right.$ EvoHGP $\left.+\mathrm{C}+\mathrm{M}_{0.5}\right)$ improves the overall performance of the algorithm. Moreover, we see that using both re-

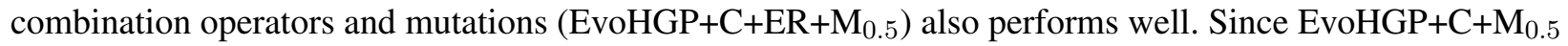
and EvoHGP $+\mathrm{C}+\mathrm{ER}+\mathrm{M}_{0.5}$ show the best convergence behavior, we restrict ourselves to these configurations for the remaining experiments performed in the paper.

\subsection{Evaluation}

We now switch to our large benchmark set to evaluate the performance of the different algorithms under consideration. Table 1 and Figure 3 compare the performance of our memetic algorithms with repeated executions of KaHyPar-CA and KaHyPar-CA-V. When looking at convergence plots, note that KaHyPar$\mathrm{CA}-\mathrm{V}$ starts later than all other algorithms and has an initially better solution quality. This is due to the fact it uses up to $100 \mathrm{~V}$-cycles before reporting the first solution. The improvements of our memetic algorithms increase with increasing $k$. This is expected as the search space of possible partitionings increases with the number of blocks. Looking at Table 1, we see that both memetic algorithms on average outperform the best partitioner currently available (KaHyPar-CA), culminating in an improvement of $5.4 \%$ for $k=128$. Furthermore both EvoHGP $+\mathrm{C}+\mathrm{M}_{0.5}$ and EvoHGP $+\mathrm{C}+\mathrm{ER}+\mathrm{M}_{0.5}$ are able to improve upon the new $\mathrm{V}$-cycling version KaHyPar-CA-V for all values of $k$ and performs $3 \%$ better on average than KaHyPar-CA-V for $k \geq 32$. While the difference in solution quality between both memetic algorithms is small on average, a Wilcoxon matched pairs signed rank test [61] (using a 1\% significance level) reveals that the improved solution quality of EvoHGP+C+ER+M 0.5 is statistically significant $(Z=-2.992857, p=0.002763795)$. This is also confirmed by the performance plot shown in Figure 4 (left). 
Table 1: Average improvement in connectivity metric over both KaHyPar-CA and the new version KaHyParCA-V.

\begin{tabular}{|c|c|c|c|c|}
\hline \multirow[b]{2}{*}{$k$} & \multicolumn{2}{|c|}{ KaHyPar-CA vs. EvoHGP } & \multicolumn{2}{|c|}{ KaHyPar-CA-V vs. EvoHGP } \\
\hline & $+\mathrm{C}+\mathrm{M}_{0.5}$ & $+\mathrm{C}+\mathrm{ER}+\mathrm{M}_{0.5}$ & $+\mathrm{C}+\mathrm{M}_{0.5}$ & $+\mathrm{C}+\mathrm{ER}+\mathrm{M}_{0.5}$ \\
\hline all & $3.3 \%$ & $3.4 \%$ & $2.3 \%$ & $2.4 \%$ \\
\hline 2 & $0.9 \%$ & $0.9 \%$ & $0.3 \%$ & $0.4 \%$ \\
\hline 4 & $1.3 \%$ & $1.4 \%$ & $0.8 \%$ & $1.0 \%$ \\
\hline 8 & $2.7 \%$ & $2.9 \%$ & $1.9 \%$ & $2.0 \%$ \\
\hline 16 & $3.5 \%$ & $3.6 \%$ & $2.5 \%$ & $2.6 \%$ \\
\hline 32 & $4.3 \%$ & $4.6 \%$ & $3.2 \%$ & $3.5 \%$ \\
\hline 64 & $4.9 \%$ & $5.0 \%$ & $3.5 \%$ & $3.6 \%$ \\
\hline 128 & $5.4 \%$ & $5.4 \%$ & $3.7 \%$ & $3.7 \%$ \\
\hline
\end{tabular}
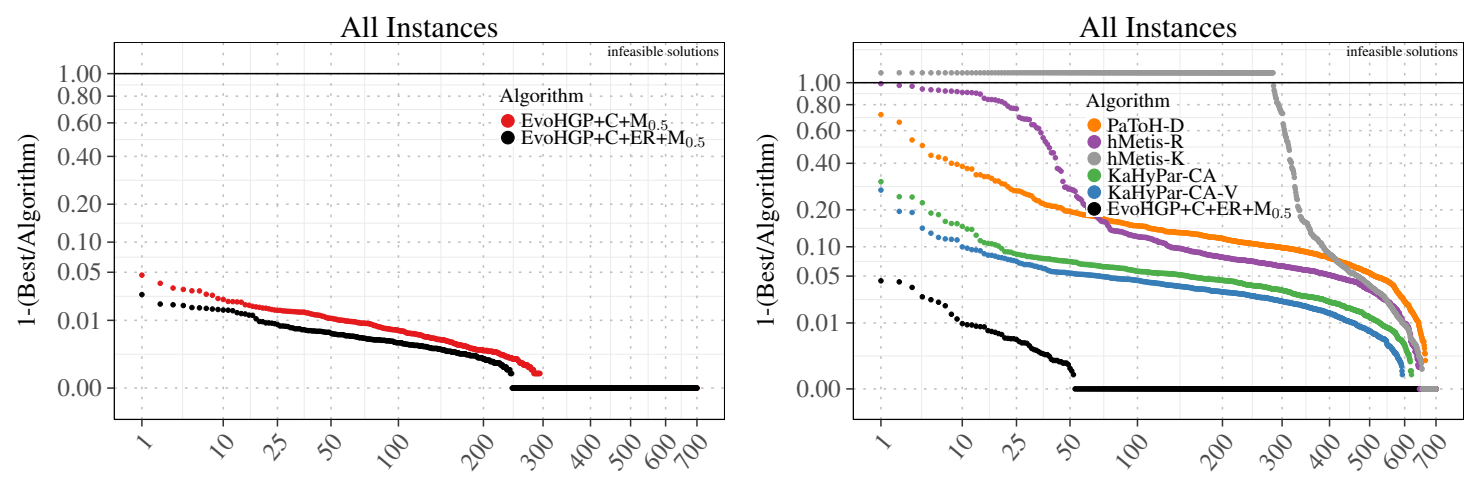

Fig. 4: Performance plots comparing the two final configurations of our evolutionary algorithm (left) and the best configuration of EvoHGP to the strongest non-evolutionary algorithms (right). EvoHGP+C+ER+M 0.5 outperforms the non-evolutionary algorithms on almost all instances.

Looking at the performance plot that compares the strongest non-evolutionary algorithms with the strongest memetic configuration in Figure 4 (right), we see that EvoHGP+C+ER+M 0.5 performs significantly better than all other algorithms. It produces the best partitions for 648 of the 700 instances. It is followed by KaHyPar-CA-V (107), KaHyPar-CA (79), hMetis-R (55), hMetis-K (46), and PaToH-D (35). Note that for some instances, multiple partitioners computed the same solution. Comparing the best solutions of EvoHGP+C+ER+M $\mathrm{M}_{0.5}$ to each partitioner individually, it produced better partitions than PaToH-D, hMetis-K, hMetis-R, KaHyPar-CA, KaHyPar-CA-V in 661, 644, 636, 609, 585 cases, respectively.

This shows that even a large number of repeated executions helps only partially to explore the huge space of possible partitionings. By combining effective exploration (global search) with exploitation (in our case using powerful $n$-level HGP algorithms) our memetic algorithm can effectively help to break out of local minima and hence explore the global solution space more extensively.

\section{Conclusion and Future Work}

EvoHGP is the first multilevel memetic algorithm to tackle the balanced hypergraph partitioning problem. Key components of our contribution are new effective multilevel recombine and mutation operations that 
incorporate information about the best solutions in the coarsening process and provide a large amount of diversity. Experiments comparing EvoHGP with a V-cycling version of KaHyPar-CA and the well known HGP tools hMetis and PaToH indicate that our evolutionary algorithm computes by far the best solutions on almost all instances. This confirms our conjecture that previous attempts to solve the HGP problem using memetic algorithms failed to be competitive with state-of-the-art tools because (i) only flat partitioning algorithms were used to drive the exploitation phase and (ii) evolutionary operators were problem agnostic and thus did not incorporate enough structural information into the algorithm. We therefore believe that EvoHGP is helpful in a wide area of application areas in which solution quality is of major importance. In the future, it would be interesting to apply EvoHGP in such application areas and to try other domain specific recombine operators that offer more specific knowledge of the application domain. In addition, it may be worth to investigate shared-memory parallelization as in [8] or a distributed memory parallelization based on islands as in [50]. Lastly, we plan to integrate our algorithm in the KaHyPar framework. 


\section{References}

1. S. N. Adya, M. C. Yildiz, I. L. Markov, P. G. Villarrubia, P. N. Parakh, and P. H. Madden. Benchmarking for large-scale placement and beyond. IEEE Transactions on Computer-Aided Design of Integrated Circuits and Systems, 23(4):472-487, April 2004.

2. Y. Akhremtsev, T. Heuer, P. Sanders, and S. Schlag. Engineering a direct $k$-way hypergraph partitioning algorithm. In 19th Workshop on Algorithm Engineering and Experiments, (ALENEX), pages 28-42, 2017.

3. C. J. Alpert. The ISPD98 Circuit Benchmark Suite. In Proceedings of the 1998 International Symposium on Physical Design, pages 80-85. ACM, 1998.

4. C. J. Alpert, J.-H. Huang, and A. B. Kahng. Multilevel Circuit Partitioning. IEEE Transactions on Computer-Aided Design of Integrated Circuits and Systems, 17(8):655-667, 1998.

5. C. J. Alpert and A. B. Kahng. Recent Directions in Netlist Partitioning: a Survey. Integration, the VLSI Journal, 19(1-2):1 - 81, 1995.

6. S. Areibi. An Integrated Genetic Algorithm With Dynamic Hill Climbing for VLSI Circuit Partitioning. In Genetic and Evolutionary Computation Conference (GECCO), pages 97-102, 2000.

7. S. Areibi and Z. Yang. Effective Memetic Algorithms for VLSI Design = Genetic Algorithms + Local Search + Multi-Level Clustering. Evolutionary Computation, 12(3):327-353, 2004.

8. E. Armstrong, G. W. Grewal, S. Areibi, and G. Darlington. An investigation of parallel memetic algorithms for VLSI circuit partitioning on multi-core computers. In Proceedings of the 23rd Canadian Conference on Electrical and Computer Engineering, CCECE, pages 1-6. IEEE, 2010.

9. C. Aykanat, B. B. Cambazoglu, and B. Uçar. Multi-level Direct K-way Hypergraph Partitioning with Multiple Constraints and Fixed Vertices. Journal of Parallel and Distributed Computing, 68(5):609-625, 2008.

10. T. Bäck. Evolutionary algorithms in theory and practice : evolution strategies, evolutionary programming, genetic algorithms. $\mathrm{PhD}$ thesis, 1996.

11. D. A. Bader, H. Meyerhenke, P. Sanders, and D. Wagner, editors. Proc. Graph Partitioning and Graph Clustering - 10th DIMACS Implementation Challenge Workshop, volume 588 of Contemporary Mathematics. AMS, 2013.

12. A. Belov, D. Diepold, M. Heule, and M. Järvisalo. The SAT Competition 2014. http://www. satcompetition.org/ $2014 / 2014$.

13. U. Benlic and J. K. Hao. A Multilevel Memetic Approach for Improving Graph $k$-Partitions. IEEE Transactions on Evolutionary Computation, 15(5):624-642, 2011.

14. T. Blickle and L. Thiele. A Comparison of Selection Schemes used in Evolutionary Algorithms. Evolutionary Computation, 4(4):361-394, 1996.

15. T. N. Bui and B. R. Moon. A Fast and Stable Hybrid Genetic Algorithm for the Ratio-Cut Partitioning Problem on Hypergraphs. In Proceedings of the 31st Conference on Design Automation, pages 664-669, 1994.

16. A. Buluç, H. Meyerhenke, I. Safro, P. Sanders, and C. Schulz. Recent Advances in Graph Partitioning. In Algorithm Engineering - Selected Results and Surveys, pages 117-158. Springer, 2016.

17. Ü. V. Catalyürek and C. Aykanat. Hypergraph-Partitioning-Based Decomposition for Parallel Sparse-Matrix Vector Multiplication. IEEE Transactions on Parallel and Distributed Systems, 10(7):673-693, Jul 1999.

18. P. Chardaire, M. Barake, and G. P. McKeown. A PROBE-Based Heuristic for Graph Partitioning. IEEE Transactions on Computers, 56(12):1707-1720, 2007.

19. J. Cohoon, J. Kairo, and J. Lienig. Evolutionary Algorithms for the Physical Design of VLSI Circuits, pages 683-711. Springer, 2003.

20. N. J. Cox. Stata tip 96: Cube roots. Stata Journal, 11(1):149-154(6), 2011. URL: http://www.stata-journal.com/ article.html?article=st0223

21. T. A. Davis and Y. Hu. The University of Florida Sparse Matrix Collection. ACM Transactions on Mathematical Software, 38(1):1:1-1:25, 2011.

22. K. A. De Jong. Evolutionary computation - a unified approach. MIT Press, 2006.

23. D. Delling, A. V. Goldberg, I. Razenshteyn, and R. F. Werneck. Graph Partitioning with Natural Cuts. In Proceedings of the 25th International Parallel and Distributed Processing Symposium, pages 1135-1146, 2011.

24. M. Deveci, K. Kaya, B. Uçar, and Ü V. Çatalyürek. Hypergraph partitioning for multiple communication cost metrics: Model and methods. Journal of Parallel and Distributed Computing, 77:69-83, 2015.

25. K. D. Devine, E. G. Boman, R. T. Heaphy, R. H. Bisseling, and Ü. V. Catalyürek. Parallel Hypergraph Partitioning for Scientific Computing. In 20th International Conference on Parallel and Distributed Processing, IPDPS, pages 124-124. IEEE, 2006.

26. W.E. Donath. Logic partitioning. Physical Design Automation of VLSI Systems, pages 65-86, 1988.

27. T. A. Feo, M. G. C. Resende, and S. H. Smith. A Greedy Randomized Adaptive Search Procedure for Maximum Independent Set. Operations Research, 42(5):860-878, 1994.

28. C.M. Fiduccia and R.M. Mattheyses. A linear-time heuristic for improving network partitions. In 19th Conference on Design Automation, pages 175-181, June 1982.

29. David E. Goldberg. Genetic algorithms in search, optimization, and machine learning. Addison-Wesley, 1989.

30. T. Heuer and S. Schlag. Improving Coarsening Schemes for Hypergraph Partitioning by Exploiting Community Structure. In 16th International Symposium on Experimental Algorithms, (SEA), page 21:121:19, 2017.

31. J. H. Holland. Adaptation in Natural and Artificial Systems. University of Michigan Press, 1975. second edition, 1992.

32. T. C. Hu and K. Moerder. Multiterminal Flows in a Hypergraph. In T.C. Hu and E.S. Kuh, editors, VLSI Circuit Layout: Theory and Design, chapter 3, pages 87-93. IEEE Press, 1985. 
33. M. Hulin. Circuit partitioning with genetic algorithms using a coding scheme to preserve the structure of a circuit, pages 75-79. Springer, 1991.

34. I. Kabiljo, B. Karrer, M. Pundir, S. Pupyrev, A. Shalita, A. Presta, and Y. Akhremtsev. Social Hash Partitioner: A Scalable Distributed Hypergraph Partitioner. pages 1-23, 2017. arXiv:1707.06665

35. G. Karypis, R. Aggarwal, V. Kumar, and S. Shekhar. Multilevel Hypergraph Partitioning: Applications in VLSI Domain. IEEE Transactions on Very Large Scale Integration VLSI Systems, 7(1):69-79, 1999.

36. G. Karypis and V. Kumar. Multilevel $K$-way Hypergraph Partitioning. In Proceedings of the 36th ACM/IEEE Design Automation Conference, pages 343-348. ACM, 1999.

37. J. Kim, I. Hwang, Y. H. Kim, and B. R. Moon. Genetic Approaches for Graph Partitioning: A Survey. In Proceedings of the 13th Annual Genetic and Evolutionary Computation Conference (GECCO), pages 473-480. ACM, 2011.

38. J.-P. Kim, Y.-H. Kim, and B.-R. Moon. A Hybrid Genetic Approach for Circuit Bipartitioning, pages 1054-1064. Springer, 2004.

39. Y.-H. Kim and B. R. Moon. Lock-Gain Based Graph Partitioning. Journal of Heuristics, 10(1):37-57, 2004.

40. T. Lengauer. Combinatorial Algorithms for Integrated Circuit Layout. John Wiley \& Sons, Inc., 1990.

41. Z. Mann and P. Papp. Formula partitioning revisited. In Daniel Le Berre, editor, POS-14. Fifth Pragmatics of SAT workshop, volume 27 of EPiC Series in Computing, pages 41-56. EasyChair, 2014.

42. P. Moscato. On Evolution, Search, Optimization, Genetic Algorithms and Martial Arts: Towards Memetic Algorithms. Technical Report C3P Report 826, California Institute of Technology, 1989.

43. P. Moscato and C. Cotta. A Modern Introduction to Memetic Algorithms, pages 141-183. Springer US, 2010.

44. D. A. Papa and I. L. Markov. Hypergraph Partitioning and Clustering. In T. F. Gonzalez, editor, Handbook of Approximation Algorithms and Metaheuristics. Chapman and Hall/CRC, 2007.

45. N. J. Radcliffe and P. D. Surry. Formal Memetic Algorithms. In T. C. Fogarty, editor, Evolutionary Computing, AISB Workshop, volume 865 of Lecture Notes in Computer Science, pages 1-16. Springer, 1994.

46. K. Roberts and Preas B. MCNC. Technical report, Physical Design Workshop, 1987.

47. Y. Saab and V. B. Rao. An Evolution-Based Approach to Partitioning ASIC Systems. In D. E. Thomas, editor, Proceedings of the 26th ACM/IEEE Design Automation Conference, pages 767-770. ACM Press, 1989.

48. L. A. Sanchis. Multiple-way Network Partitioning. IEEE Transactions on Computers, 38(1):62-81, 1989.

49. P. Sanders and C. Schulz. Engineering Multilevel Graph Partitioning Algorithms. In Algorithms - ESA, volume 6942 of LNCS, pages 469-480. Springer, 2011.

50. P. Sanders and C. Schulz. Distributed Evolutionary Graph Partitioning. In 12th Workshop on Algorithm Engineering and Experimentation (ALENEX), pages 16-29, 2012.

51. P. Sanders and C. Schulz. Think Locally, Act Globally: Highly Balanced Graph Partitioning. In 12th International Symposium on Experimental Algorithms (SEA), volume 7933 of LNCS, pages 164-175. Springer, 2013.

52. S. Schlag, V. Henne, T. Heuer, H. Meyerhenke, P. Sanders, and C. Schulz. $k$-way Hypergraph Partitioning via $n$-Level Recursive Bisection. In 18th Workshop on Algorithm Engineering and Experiments (ALENEX), pages 53-67, 2016.

53. A. J. Soper, C. Walshaw, and M. Cross. A Combined Evolutionary Search and Multilevel Optimisation Approach to GraphPartitioning. Journal of Global Optimization, 29(2):225-241, 2004.

54. A. Trifunovic. Parallel Algorithms for Hypergraph Partitioning. $\mathrm{PhD}$ thesis, University of London, 2006.

55. A. Trifunovic and W. J. Knottenbelt. Parallel Multilevel Algorithms for Hypergraph Partitioning. Journal of Parallel and Distributed Computing, 68(5):563 - 581, 2008.

56. Ü. V. Çatalyürek and M. Deveci and K. Kaya and B. Uçar. UMPa: A multi-objective, multi-level partitioner for communication minimization. In Bader et al. [11], pages 53-66.

57. B. Vastenhouw and R. H. Bisseling. A Two-Dimensional Data Distribution Method for Parallel Sparse Matrix-Vector Multiplication. SIAM Review, 47(1):67-95, 2005.

58. N. Viswanathan, C. Alpert, C. Sze, Z. Li, and Y. Wei. The DAC 2012 Routability-driven Placement Contest and Benchmark Suite. In 49th Annual Design Automation Conference, DAC, pages 774-782. ACM, 2012.

59. C. Walshaw. Multilevel Refinement for Combinatorial Optimisation Problems. Annals of Operations Research, 131(1-4):325372,2004

60. S. Wichlund and E. Aas. On Multilevel Circuit Partitioning. In Proceedings of theIEEE/ACM International Conference on Computer-Aided Design (ICCAD), pages 505-511, 1998.

61. F. Wilcoxon. Individual Comparisons by Ranking Methods. Biometrics Bulletin, 1(6):80-83, 1945. 


\section{A Benchmark Hypergraphs}

Table 2: Basic properties of our small benchmark subset.

\begin{tabular}{|c|c|c|c|c|c|c|c|}
\hline Hypergraph & $n$ & $m$ & $p$ & Hypergraph & $n$ & $m$ & $p$ \\
\hline \multicolumn{4}{|c|}{ ISPD98 } & \multicolumn{4}{|c|}{ SAT14Primal } \\
\hline ibm06 & 32498 & 34826 & 128182 & $6 s 153$ & 85646 & 245440 & 572692 \\
\hline ibm07 & 45926 & 48117 & 175639 & aaai10-planning-ipc5 & 53919 & 308235 & 690466 \\
\hline ibm08 & 51309 & 50513 & 204890 & atco_enc2_opt1_05_21 & 56533 & 526872 & 2097393 \\
\hline ibm09 & 53395 & 60902 & 222088 & dated-10-11-u & 141860 & 629461 & 1429872 \\
\hline ibm10 & 69429 & 75196 & 297567 & hwmcc10-timeframe & 163622 & 488120 & 1138944 \\
\hline \multicolumn{4}{|c|}{ SAT14Dual } & \multicolumn{4}{|c|}{ SPM } \\
\hline $6 s 133$ & 140968 & 48215 & 328924 & laminar_duct3D & 67173 & 67173 & 3833077 \\
\hline $6 \mathrm{~s} 153$ & 245440 & 85646 & 572692 & mixtank_new & 29957 & 29957 & 1995041 \\
\hline $6 \mathrm{~s} 9$ & 100384 & 34317 & 234228 & mult_dcop_01 & 25187 & 25187 & 193276 \\
\hline dated-10-11-u & 629461 & 141860 & 1429872 & RFdevice & 74104 & 74104 & 365580 \\
\hline dated-10-17-u & 1070757 & 229544 & 2471122 & vibrobox & 12328 & 12328 & 342828 \\
\hline \multicolumn{4}{|c|}{ SAT14Literal } & & & & \\
\hline $6 s 133$ & 96430 & 140968 & 328924 & & & & \\
\hline $6 s 153$ & 171292 & 245440 & 572692 & & & & \\
\hline aaai10-planning-ipc5 & 107838 & 308235 & 690466 & & & & \\
\hline atco_enc2_opt1_05_21 & 112732 & 526872 & 2097393 & & & & \\
\hline dated-10-11-u & 283720 & 629461 & 1429872 & & & & \\
\hline
\end{tabular}


Table 3: Basic properties of our large benchmark subset.

\begin{tabular}{|c|c|c|c|c|c|c|c|}
\hline \multirow[t]{2}{*}{ Hypergraph } & $n$ & $m$ & $p$ & Hypergraph & $n$ & $m$ & $p$ \\
\hline & \multicolumn{3}{|l|}{ DAC2012 } & \multicolumn{4}{|c|}{ SAT14Primal } \\
\hline superblue19 & 522482 & 511685 & 1713796 & AProVE07-27 & 7729 & 29194 & 77124 \\
\hline superblue 16 & 698339 & 697458 & 2280417 & countbitssrl032 & 18607 & 55724 & 130020 \\
\hline superblue14 & 630802 & 619815 & 2048903 & $6 \mathrm{~s} 184$ & 33365 & 97516 & 227536 \\
\hline superblue3 & 917944 & 898001 & 3109446 & $6 s 9$ & 34317 & 100384 & 234228 \\
\hline \multicolumn{4}{|c|}{ ISPD98 } & $6 s 133$ & 48215 & 140968 & 328924 \\
\hline ibm09 & 53395 & 60902 & 222088 & $6 s 153$ & 85646 & 245440 & 572692 \\
\hline ibm11 & 70558 & 81454 & 280786 & atco_enc1_opt2_10_16 & 9643 & 152744 & 641139 \\
\hline ibm 10 & 69429 & 75196 & 297567 & aaai10-planning-ipc5 & 53919 & 308235 & 690466 \\
\hline ibm12 & 71076 & 77240 & 317760 & hwmcc10-timeframe & 163622 & 488120 & 1138944 \\
\hline ibm13 & 84199 & 99666 & 357075 & itox_vc1130 & 152256 & 441729 & 1143974 \\
\hline ibm14 & 147605 & 152772 & 546816 & dated-10-11-u & 141860 & 629461 & 1429872 \\
\hline ibm 15 & 161570 & 186608 & 715823 & atco_enc1_opt2_05_4 & 14636 & 386163 & 1652800 \\
\hline ibm16 & 183484 & 190048 & 778823 & manol-pipe-g10bid_i & 266405 & 792175 & 1848407 \\
\hline ibm 18 & 210613 & 201920 & 819697 & manol-pipe-c8nidw & 269048 & 799867 & 1866355 \\
\hline ibm 17 & 185495 & 189581 & 860036 & atco_enc2_opt1_05_21 & 56533 & 526872 & 2097393 \\
\hline \multicolumn{4}{|c|}{ SAT14Dual } & dated-10-17-u & 229544 & 1070757 & 2471122 \\
\hline AProVE07-27 & 29194 & 7729 & 77124 & ACG-20-5p0 & 324716 & 1390931 & 3269132 \\
\hline countbitssrl032 & 55724 & 18607 & 130020 & ACG-20-5p1 & 331196 & 1416850 & 3333531 \\
\hline $6 s 184$ & 97516 & 33365 & 227536 & \multicolumn{4}{|c|}{ SPM } \\
\hline $6 s 9$ & 100384 & 34317 & 234228 & powersim & 15838 & 15838 & 67562 \\
\hline $6 s 133$ & 140968 & 48215 & 328924 & as-caida & 31379 & 26475 & 106762 \\
\hline $6 s 153$ & 245440 & 85646 & 572692 & hvdc1 & 24842 & 24842 & 159981 \\
\hline atco_enc1_opt2_10_16 & 152744 & 9643 & 641139 & Ill_Stokes & 20896 & 20896 & 191368 \\
\hline aaai10-planning-ipc5 & 308235 & 53919 & 690466 & mult_dcop_01 & 25187 & 25187 & 193276 \\
\hline hwmcc10-timeframe & 488120 & 163622 & 1138944 & lp_pds_20 & 108175 & 33798 & 232647 \\
\hline itox_vc1130 & 441729 & 152256 & 1143974 & lhr14 & 14270 & 14270 & 307858 \\
\hline dated-10-11-u & 629461 & 141860 & 1429872 & $c-61$ & 43618 & 43618 & 310016 \\
\hline atco_enc1_opt2_05_4 & 386163 & 14636 & 1652800 & ckt11752_dc_1 & 49702 & 49702 & 333029 \\
\hline manol-pipe-g10bid_i & 792175 & 266405 & 1848407 & RFdevice & 74104 & 74104 & 365580 \\
\hline manol-pipe-c8nidw & 799867 & 269048 & 1866355 & light_in_tissue & 29282 & 29282 & 406084 \\
\hline atco_enc2_opt1_05_21 & 526872 & 56533 & 2097393 & Pres_Poisson & 14822 & 14822 & 715804 \\
\hline dated-10-17-u & 1070757 & 229544 & 2471122 & Andrews & 60000 & 60000 & 760154 \\
\hline ACG-20-5p0 & 1390931 & 324716 & 3269132 & 2D_54019_highK & 54019 & 54019 & 996414 \\
\hline ACG-20-5p1 & 1416850 & 331196 & 3333531 & case 39 & 40216 & 40216 & 1042160 \\
\hline \multicolumn{4}{|c|}{ SAT14Literal } & denormal & 89400 & 89400 & 1156224 \\
\hline AProVE07-27 & 15458 & 29194 & 77124 & 2cubes_sphere & 101492 & 101492 & 1647264 \\
\hline countbitssrl032 & 37213 & 55724 & 130020 & av41092 & 41092 & 41092 & 1683902 \\
\hline $6 s 184$ & 66730 & 97516 & 227536 & Lin & 256000 & 256000 & 1766400 \\
\hline $6 s 9$ & 68634 & 100384 & 234228 & $\operatorname{cfd} 1$ & 70656 & 70656 & 1828364 \\
\hline $6 s 133$ & 96430 & 140968 & 328924 & opt1 & 15449 & 15449 & 1930655 \\
\hline $6 s 153$ & 171292 & 245440 & 572692 & mixtank_new & 29957 & 29957 & 1995041 \\
\hline atco_enc1_opt2_10_16 & 18930 & 152744 & 641139 & sme $3 \mathrm{Db}$ & 29067 & 29067 & 2081063 \\
\hline aaai10-planning-ipc5 & 107838 & 308235 & 690466 & mc2depi & 525825 & 525825 & 2100225 \\
\hline hwmcc10-timeframe & 327243 & 488120 & 1138944 & poisson3 $\mathrm{Db}$ & 85623 & 85623 & 2374949 \\
\hline itox_vc1130 & 294326 & 441729 & 1143974 & rgg_n_2_18_s0 & 262144 & 262141 & 3094566 \\
\hline dated-10-11-u & 283720 & 629461 & 1429872 & cnr-2000 & 325557 & 247501 & 3216152 \\
\hline atco_enc1_opt2_05_4 & 28738 & 386163 & 1652800 & $\mathrm{~m} 14 \mathrm{~b}$ & 214765 & 214765 & 3358036 \\
\hline manol-pipe-g10bid_i & 532810 & 792175 & 1848407 & laminar_duct3D & 67173 & 67173 & 3833077 \\
\hline manol-pipe-c8nidw & 538096 & 799867 & 1866355 & gearbox & 153746 & 153746 & 9080404 \\
\hline atco_enc2_opt1_05_21 & 112732 & 526872 & 2097393 & BenElechi1 & 245874 & 245874 & 13150496 \\
\hline dated-10-17-u & 459088 & 1070757 & 2471122 & af_shell1 & 504855 & 504855 & 17588875 \\
\hline ACG-20-5p0 & 649432 & 1390931 & 3269132 & & & & \\
\hline ACG-20-5p1 & 662392 & 1416850 & 3333531 & & & & \\
\hline
\end{tabular}

